\title{
Smart System for Attendance
}

\author{
Preeti Pandey ${ }^{1,}$ Dr. D. John Arvindhar ${ }^{2}$ \\ ${ }^{l}$ Student, MCA, Hindustan Institute of Technology and Science \\ Padur, Chennai, India \\ ${ }^{2}$ Department of Computer Science Engineering, Hindustan Institute of Technology and Science \\ Padur, Chennai, India \\ Email : ${ }^{1}$ ppandey1332@gmail.com, ${ }^{2}$ jaravindhar@hindustanuniv.ac.in
}

\begin{abstract}
Attendance management system is a necessary tool for taking attendance in any environment where attendance is critical. We can see most of the existing system is time consuming every time it requires human effort to take the attendance. Face recognition is an important application of Image processing owing to its use in many fields. Identification of individuals in an organization for the purpose of attendance is one such application of face recognition. Maintenance and monitoring of attendance records plays a vital role in the analysis of performance of any organization. The purpose of developing attendance management system is to computerize the traditional way of taking attendance. Automated Attendance Management System performs the daily activities of attendance marking and analysis with reduced human intervention. In our proposed system we will build an attendance system which will recognize the face of the student and check the appearance of the student face in the data archive and mark it as present or absent. Absent student information sent to their guardian by sending automatic message. as well as a mail sent to the staff describing number of student attending or missed the class. Thus, this project saves the time of the humans that will helps in saving maintenance and also generating report of absent and present student.
\end{abstract}

\section{Keywords-Deep Learning, MobileFaceNet, Face Segmentor-Enhanced Network, UltraLight Face Detector, Textlocal, Simple Mail Transfer Protocol.}

\section{INTRODUCTION}

It is estimated that managing attendance is a necessary and tedious process for the institute. Face recognize is an essential area in many places. Now in every institute calling the students name and waiting for their answers after this they are creating report to see student's performance. Every institute follows their own way for taking attendance. Some are taking attendance manually using paper or register file or biometric techniques. Attendance management facilitates information of the student in a class. The purpose of attendance system is to computerized the traditional system smartly and find the availability of the students.

Here in this project Deep learning technology is used. Deep Learning is a part of machine learning computer software that imitate the neural net of neurons in an intelligence. Deep learning is a combination of two words here the word "Deep" means quantities of invisible surface neural network.

Deep learning layers are have following layers

- Input Layer is a firstlayer

- Output Layer is the second layer

In between above two layers are called Hidden Layers are the third layer. The word deep means the network join neuronsin more than two layers.

Deep learning can exceed regular method. As compared, deep learning algorithm in image classification, face recognition and voice recognition area is found that deep learning algorithm more accurate from the machine learning algorithm. In image classification it is the accuracy of $41 \%, 27 \%$ more accurate in face recognition and in voice recognition is $25 \%$ accurate.

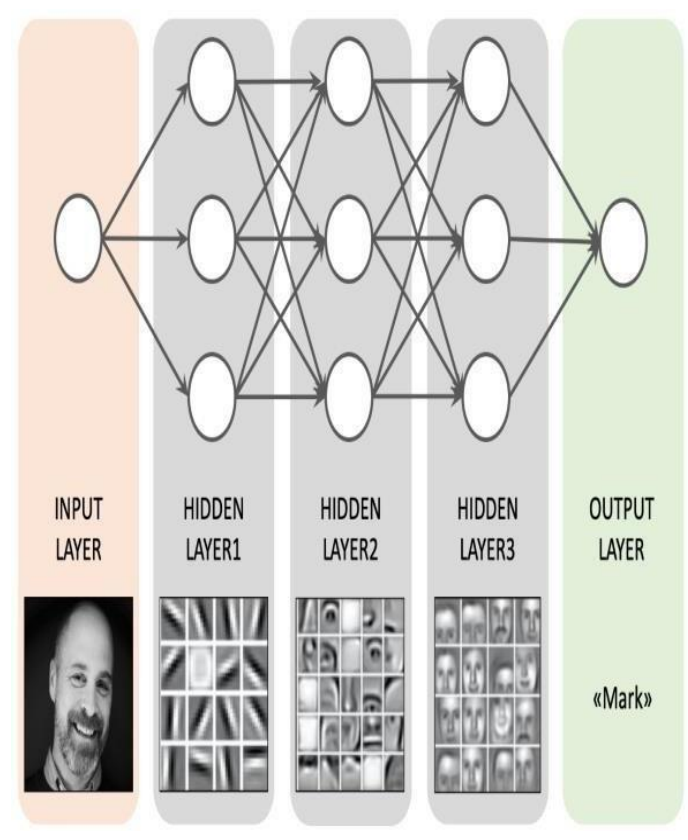

Figure 1: Deep Learning Layers

This attendance system being specially developed with point of view of school and college with respect to their economic. This system is cost effective because it is eliminating the paper work fully. The high-tech requirement for the system is profitable.

This system is not using any additional appliances i.e. hardware and application software. This system is fast working and quite easy to use for technical and nontechnical person. The interface of the system is simple and efficient therefore not any special training is requiring for operating the system. 


\section{RELATED WORD}

A Face Segmentor-Enhanced Network (FSENet) [1] for face recognition to exploit facial localized property. They presented a facial segmentor network to facial components. In this paper author obtained the pixel-level segmentation. They obtained part mask module for facial features on the basis of parsing maps. By using FSENet they have incorporated the global appearance features and local information. The advantage of this Incorporates holistic and local information to improve the discriminative power of the face descriptor.

In another research work, the novel Wasserstein convolutional neural network (WCNN) [2] approach for learning invariant features between near-infrared (NIR) and visual (VIS) face images (i.e., NIR-VIS face recognition). The low-level layers of the WCNN are trained with widely available face images in the VIS spectrum, and the highlevel layer is divided into three parts: the NIR layer, the VIS layer and the NIR-VIS shared layer. The first two layers aim at learning modality-specific features, and the NIR-VIS shared layer is designed to learn a modality-invariant feature subspace. The Wasserstein distance is introduced into the NIR-VIS shared layer to measure the dissimilarity between heterogeneous feature distributions.

In other research paper This paper developed Set-to- Set (S2S) [3] distance to measure the similar images. This paper embeds the kNN-average pooling to a deep network in the end to end fashion. Furthermore, they show that by using S2S distance they embedded metrics. S2S shows to deep learning achieve the state of the art on the result of IJB-A which based on set based face data.

This paper proposes a simultaneous feature and dictionary learning SFDL [4] method for image set based face recognition, in that many pictures are captured in different poses, expression and poses. SFDL method is used to learn discriminative, dictionaries simultaneously from raw face pixels from different face image set. this paper developed a deep simultaneous feature and dictionary learning (D-SFDL) for joining hierarchical image of the face image set. This both are the structured deep learning. they used video for face extraction and recognition and randomly selects the image set of particular person.

A new discriminative deep metric learning (DDML) [5] method for face and kinship verification in wild conditions. DDML method to train a deep neural network to learn a set of hierarchical nonlinear transformations to project face pairs into the same latent feature space, under which the distance of each positive pair is reduced and that of each negative pair is enlarges respectively.

\section{Proposed System}

To design a smart attendance system using the automatic face recognition system, send automatic message to the parent regarding the absence of the student, send automatic report to the staff through mail depending on the number of present and absent students for live monitoring. In this system we designed an attendance system which will observe the student's face and check the appearance of the face data of student in the data archive and mark it as present or absent respectively. This system using Ultralight detector and Mobilefacenet techniques to detect the face and recognize it and compare with the database. An SMS API is included in the system to automatically send SMS to student's guardian if their child missed the class or absent. Third party email sending is incorporated to send mail to the staff.

\section{ADVANTAGES:}

- Automatic attendance system using face recognition

- Saves time

- Auto report generation

- Automatic message to the parents

- Automatic mail to the staff

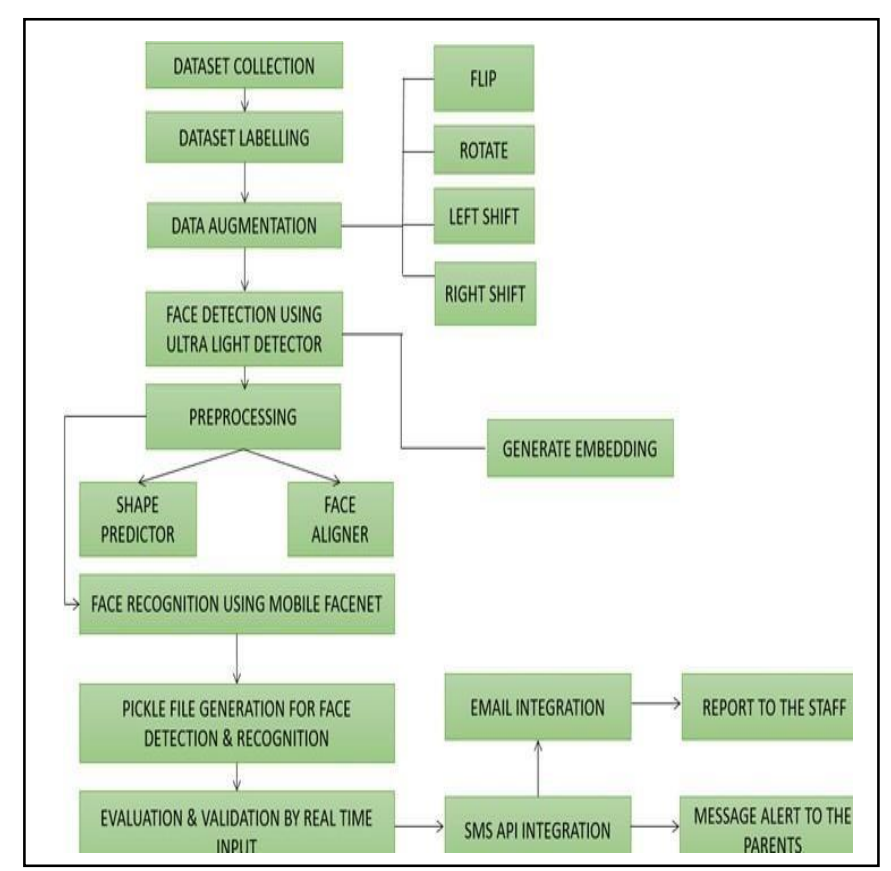

Figure 3.1: Proposed system architecture for attendance system

In introduction, Fig 3.1 shows first the Intel Processor has been configured to work on. The necessary libraries needed for the system are installed using the command window which is This system is using command window for installing the packages as well as for running the system project. The datasets for the student attendance is been collected by taking the videos of the students. Now the datasets are trained by the Intel Processor using the deep 
learning algorithm to identify the correct face and mark the student the availability of the student in class automatically. Now the existing Intel processor is qualified to catch faces trained by it, it is switched on and the camera is being initiated.

The live streaming from the camera gets started and whenever faces come in front of the camera it is detected and the features are produced.

Here MobileFaceNet and Ultra-light is used for face recognition during the live streaming of the camera. Asthe students face matches with any of the face in the trained dataset, the student is marked present. And the process goes on until the attendance time closes. As the attendance time closed, automatically the messages are sent to the parent whose children haven't attended the class. At the end automatically a report is created and mailed to staff of the related class where staff can have a record of the class attendance. Thus, this system helps in effective implementation of automatic attendance system using the latest face recognition technology as well as saves time of the institution.

\section{A. Dataset collection Module}

In computer vision tasks deep Learning definitely by far the perfect performing method for solving many challenging real world problems.

These Deep Learning mechanism that have been operating so strong need material- lots of material; that material is data. The more labelled data we have, the better our model performs. The higher labelled data we can have, the better our system will perform. This huge amount of data idea leading to higher performance explored by Google at a large scale with a 300 Million images of dataset!

In this system we have collected the dataset by capturing a short video of a person such that it will be converted into images to from dataset.

\section{B. Data Augmentation Module}

Deep Learning neural network performance depends on the amount of data it will improve as amount of data available. Data augmentation is a procedure to naturally make new training data from existing data.

This is completed by implementing domain-specific techniques that create new and different training examples from the training data.

The well-known type of data augmentation is images augmentation and associates making converted versions of pictures in the dataset that exist to the equal class as the original picture.

Convert of pictures include a range of processes from the field of image direction, like shifts, flips and zooms etc.

\section{Object Detection Module:}

The "Ultra-Light-Fast-Generic-Face-Detector" is designed for general-purpose detection for face applications in low-light devices and smart phones as well personal computer (CPU and GPU). These model is a real- time ultra-lightweight universal detection for face model built for edge computing tools or low-power devices. It can be used in low-power computing devices.

\section{Features}

- In terms of model size, the default FP32 precision (pth) file size is $1.04 \sim 1.1 \mathrm{MB}$, and the inference frame int 8 is about $300 \mathrm{~KB}$.

- In the calculation of the model, the input resolution of 320x240 is about 90 109 M-Flops.

- There are two versions of the model, version-slim (slightly faster simplification), version-RFB (with the modified RFB module, higher precision).

- Provides pre-training models using wider face training at 320x240 and 640x480 different input resolutions to better work in different application scenarios.

- Support for onxx export, easy to transplant.

\section{Face Recognition Module:}

For recognizing the face this system using mobile facenet which is a more accurate in classifying face. MobileFaceNet is a neural network and obtains accuracy upto 99.28 percent on labelled faces in the wild (LFW) dataset, and a 93.05 percent accuracy on recognising faces in the AgeDB dataset. The network used around a million parameters taking only 24 milliseconds to run and produce results on a Qualcomm Snapdragon processor.

The remarkable achievement is that MobileFaceNets achieves comparable accuracy with very small budget.

The detailed architecture is mentioned in the table below:

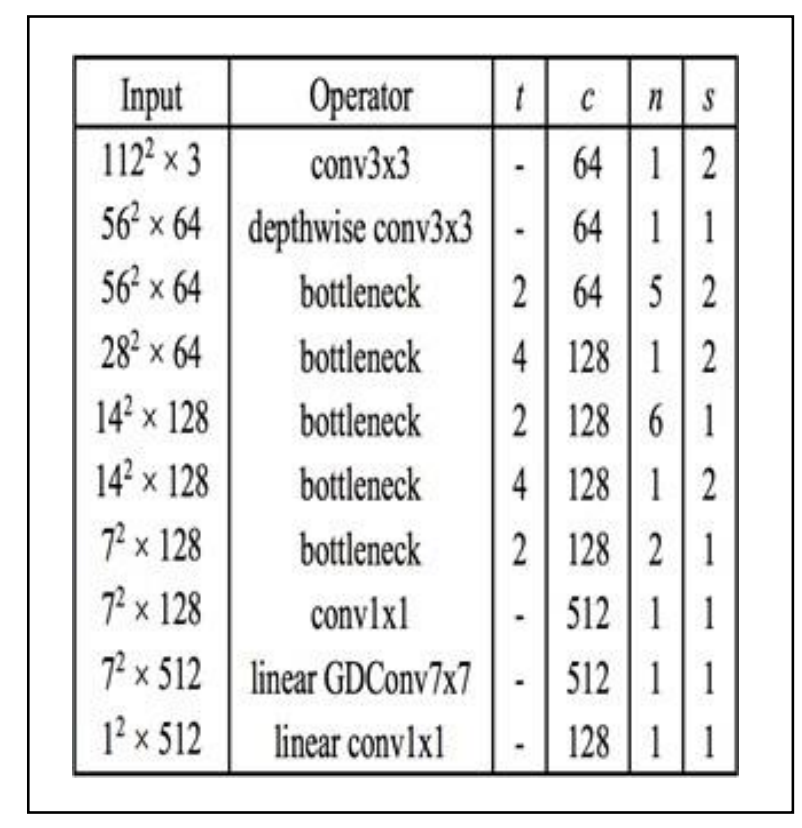

Figure 3.2: MobileFaceNet architecture 


\section{E. SMS API Integration Module:}

A well-defined software for sending SMS is SMS API. SMS API enables to send shortened message by code via SMS Gateway.

Textlocal is a free API to send and receive messages through an SMS gateway. By applying this user can create contacts, delete contacts or send a bulk SMS. We are using Textlocal SMS API for our integration which enables us to easily integrate our SMS services.

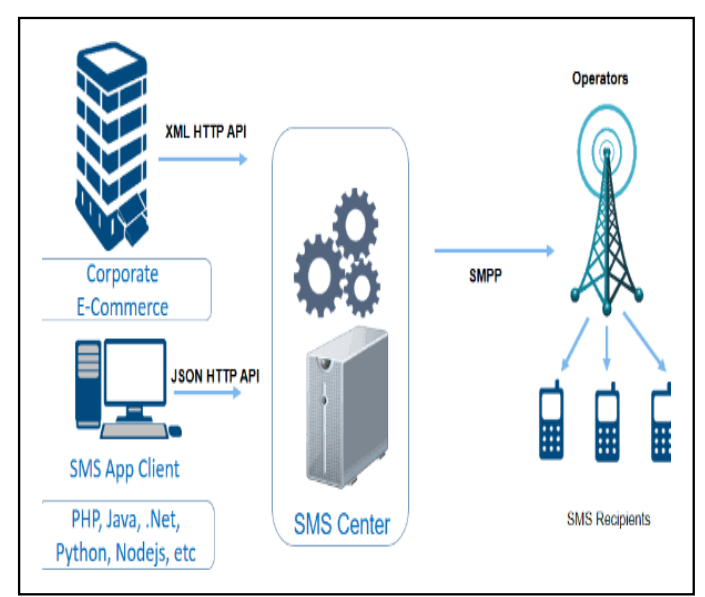

Figure 3.3: SMS API Dataflow Diagram

As the above diagram shows that framework for text message conversions and internet network mainly divided, Text message api a lot used to 'bridge the gap' from among broadcasting carrier system and network.

Text message api in the way of permit simply deliver and get text message through logic written for popular web frame. It will send SMS to parents in case of the absence of the student.

\section{F. Email integration Module:}

For integrating email system using the SMTP protocol which is used for sending and receiving mail as configured by us. The work flow of that module can be seen as follows.

Here we are using SMTP server for sending the email through Intel processor. In SMTP first session start between end user and server while MTA (Mail Transfer Agent) and MDA (Mail Delivery Agent) implement domain searching and delivery service.

TCP/IP network provides standard protocol SMTP for email services and ability to get and deliver email messages.

Python program is very useful application for getting email alerts or set of data using. By using python, we need some library i.e. smtplib in python script. These have many versions but pi is more reliable for this system. below.

An overall flow of the working can be seen in the figure

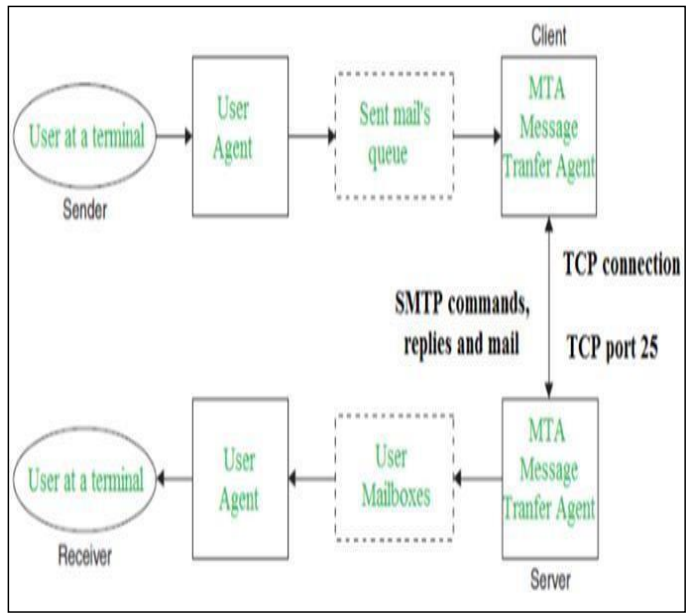

Figure 3.4: Email Integration

\section{IV.RESULT AND ANALYSIS}

In order to make the attendance system, we need to collect the dataset of the students and train them accordingly such that it will recognize the students and automatically mark the students as present or absent. The figure below, shows the dataset collected.

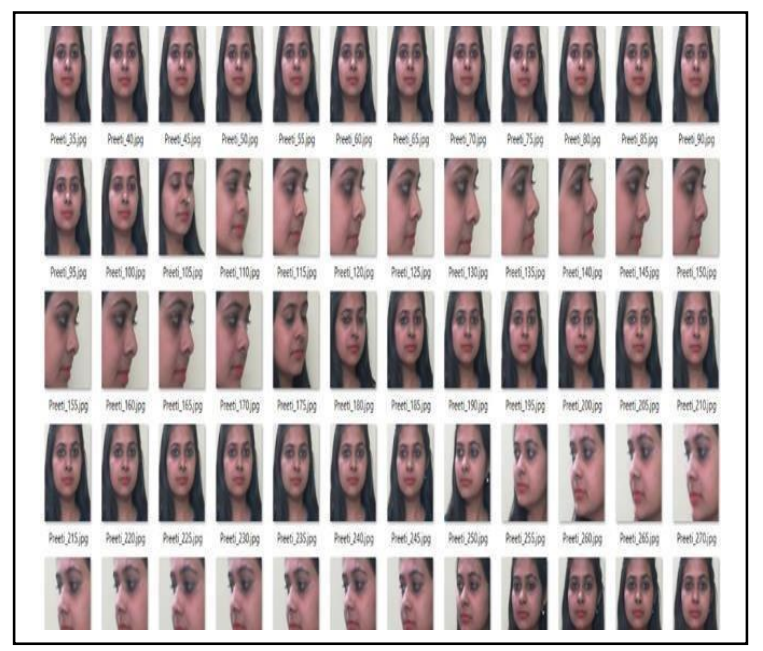

Figure 4.1: Accuracy of the training dataset

As the training gets completed. The face detection when anyone stand in front of the camera can be seen below. 


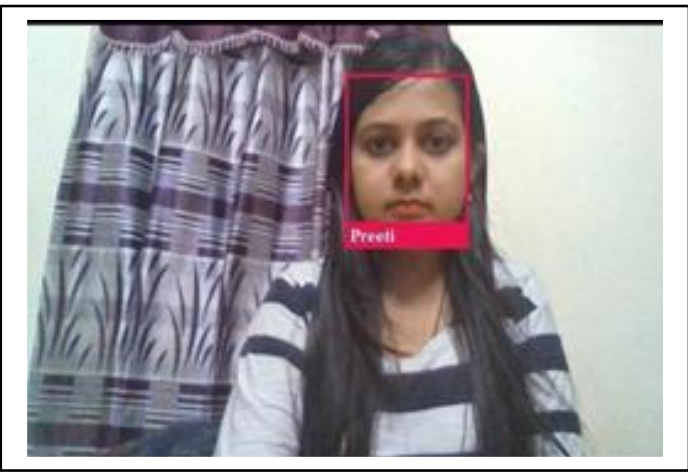

Figure 4.2: Face Detection

In Fig. 4.2. Shows the detection of face. It will detect multiple faces of students for attendance system.

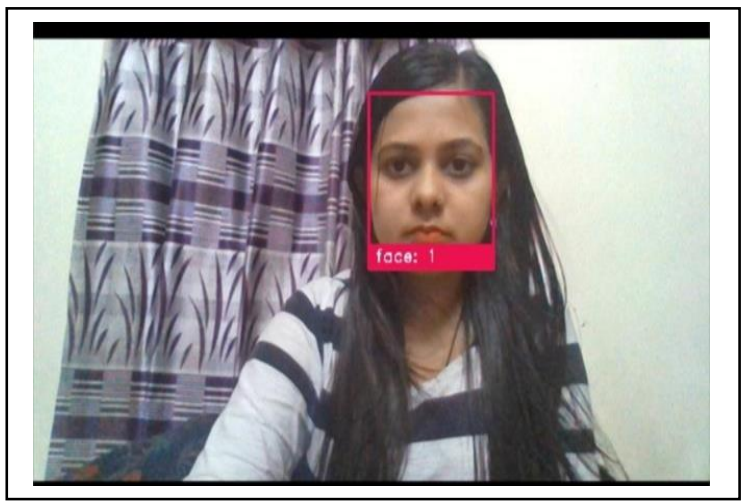

Figure 4.3: Face Recognition

Next the face recognition comes and you can be seen in the figure below which is recognize the faces of multiple students if they are in front of camera and shows their names according to their dataset.

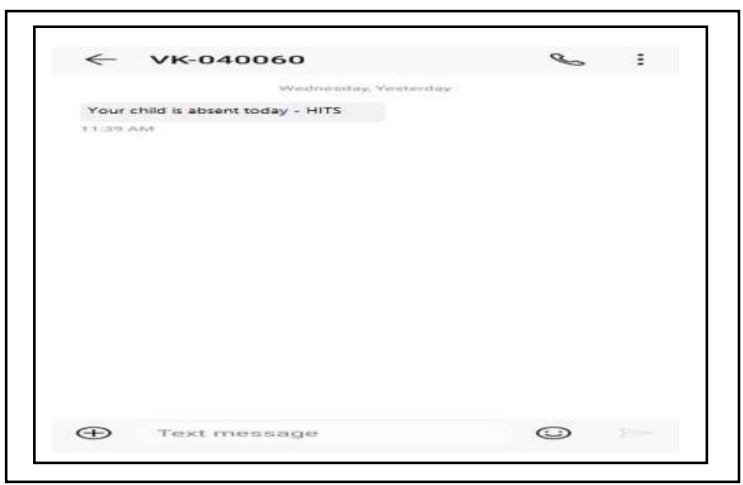

Figure 4.4: Attendance Marking

As the face recognition get completed, we can set the timing for each class such that the faces will be recognized only when they come during the class time.

Vol. 3 (12), June 2020, www.ijirase.com

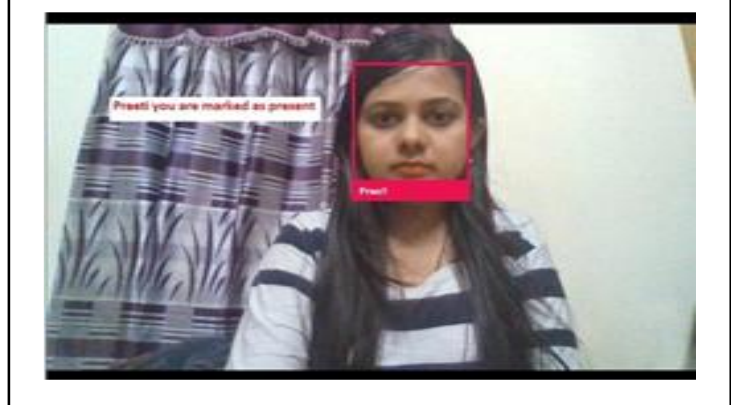

Figure 4.5: Message to parent

The above figure shows that detect face of the student and mark them as present. This system will mark the students one by one.

Whenever the student is absent an automatic message is sent to the mobile number that will provide by student's parent that can be seen in the figure below. This will be send after the class time is over.

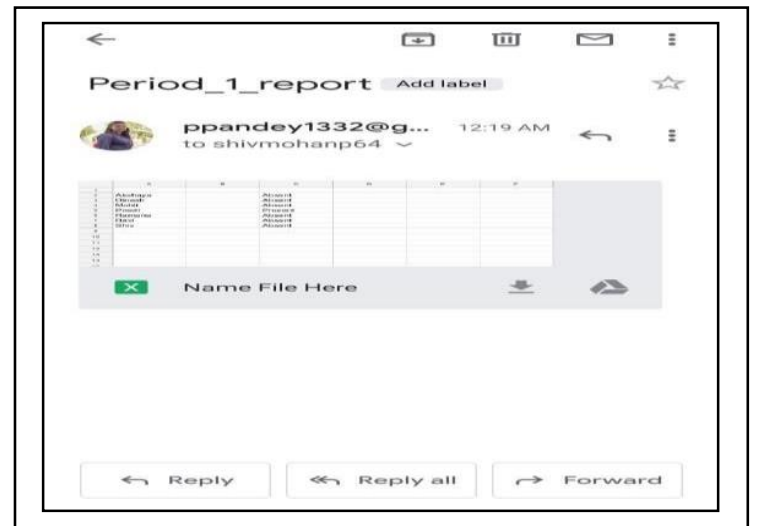

Figure 4.6: Mail Sending

Above figure shows the absent message that parent will receive on the absence of their children.The number of present and absent students are noted in excel file which is then sent as a mail to the staff mail id that is fed into the code and can be seen in the figure below

As the class timing gets over the number of students present and absent will be saved to excel sheet. Here a report is generated and send to staff mail id that can be seen in the table below.

Table 1: Absent and Present

\begin{tabular}{|l|l|}
\hline Akashaya & Absent \\
\hline Dinesh & Absent \\
\hline Mohit & Absent \\
\hline Preeti & Present \\
\hline Ramana & Absent \\
\hline Ravi & Absent \\
\hline Shiv & Absent \\
\hline
\end{tabular}

Thus this system helps in automating the attendance system which will give rise to all the attendance management 
team. It will also save the staff time.

\section{CONCLUSION AND Future Work}

Thus this project describes a project which will identify the student's face and check the appearance of the students by using their face and mark them absent or present. So this system will save the time of the person and escape physical work. It determined the absent students then an automatic message will be sent to their parents as well as Automatic mail sent to the staff also the report will generate automatically. Thus, this project helps in monitoring the student whether they attend class or not effectively.

In the coming future, we review the application of the student's smart attendance system technology and it can promote for MNC, teachers, or other private office with more accuracy compared to this project there have more chance to develop or convert project in multiple forms.

\section{REFERENCES}

[1] Cheng, Xiaojuan, et al. "Face Segmentor-Enhanced Deep Feature Learning for Face Recognition." IEEE Transactions on Biometrics, Behavior, and Identity Science 1.4 (2019): 223-237.

[2] He, Ran, et al. "Wasserstein cnn: Learning invariant features for nir-vis face recognition." IEEE transactions on pattern analysis and machine intelligence 41.7 (2018):1761-1773.

[3] Zhao, Shichao, et al. "Pooling the convolutional layers in deep convnets for video action recognition." IEEE Transactions on Circuits and Systems for Video Technology 28.8 (2017): 18391849.

[4] Lu, Jiwen, Gang Wang, and Jie Zhou. "Simultaneous feature and dictionary learning for image set based face recognition." IEEE Transactions on Image Processing 26.8 (2017): 40424054.

[5] Lu, Jiwen, Junlin $\mathrm{Hu}$, and Yap-Peng Tan. "Discriminative deep metric learning for face and kinship verification." IEEE Transactions on Image Processing 26.9 (2017): 4269-4282.

[6] Stuhlsatz, Andre, Jens Lippel, and Thomas Zielke. "Feature extraction with deep neural networks by a generalized discriminant analysis." IEEE transactions on neural networks and learning systems 23.4 (2012): 596-608.

[7] Agrawal, Aditi, et al. "Hand Down, Face Up: Innovative Mobile Attendance System Using Face Recognition Deep Learning." Proceedings of 3rd International Conference on Computer Vision and Image Processing. Springer, Singapore, 2020.

[8] Bah, Serign Modou, and Fang Ming. "An improved face recognition algorithm and its application in attendance management system." Array 5 (2020): 100014.

[9] Chintalapati, Shireesha, and M. V. Raghunadh. "Automated attendance management system based on face recognition algorithms." 2013 IEEE International Conference on Computational Intelligence and Computing Research. IEEE, 2013.

[10] Wang, Zhenzhou, et al. "A New Face Recognition Method for Intelligent Security." Applied Sciences 10.3 (2020): 852. 